\title{
The Vapor-Liquid Phase Diagram of Pure Methane Using Temperature-Dependent Interaction Parameters: A Monte Carlo Simulation
}

\author{
Ibrahim Suleiman ${ }^{1}$, Ali Al-Matar ${ }^{2}$ \\ 1) Al-Balqa Applied University, Faculty of Engineering Technology, Chemical Engineering Department, Amman, Jordan \\ ${ }^{2)}$ Chemical Engineering Department, Faculty of Engineering and Technology, University of Jordan, Amman, Jordan
}

\begin{abstract}
Adopting temperature-dependent interaction parameters in the Lennard-Jones potential, the vapor-liquid phase diagram of methane was produced using NVT Gibbs Ensemble Monte Carlo technique. Published second virial coefficient data were used to fit a simple two-parameter temperature-dependent model for the interaction parameters. The simulations were carried out in the temperature range 120-190 K. The critical density and temperature were evaluated using Ising-scaling model. Using the temperature-dependent interaction parameters in the simulation has reduced the root mean square deviation by $94.7 \%$ compared to the temperature-independent interaction parameters. The evaluated critical temperature was enhanced using temperature-dependent interaction parameters, whereas the simulations using temperature-independent interaction parameters predict a better critical density value.
\end{abstract}

Keywords: Phase diagram, TDIP, TIIP, Monte Carlo Simulation, Vapor-liquid equilibrium

\section{Introduction}

Methane, the major constituent of natural gas, represents a promising alternative energy source. It is also used to calibrate the density transducers which are utilized to directly measure the densities of natural gas transferred in pipelines. It can also be added to some refrigerants to enhance their properties (Betaouaf, et al., 2014, Fischer, et al., 1984, Li, et al., 2012, Nie, et al., 2018, Petropoulou, et al., 2018, Ungerer, et al., 2007, Uribe-Vargas and Trejo, 2005, Vrabec and Fischer, 1996). Knowledge of the thermodynamic properties of methane is necessary to processes of liquefaction, separation, and storage. Therefore, it is required to provide accurate thermodynamic properties of this alkane. Among several models available for modeling the intermolecular forces, Lennard-Jones (LJ) potential have shown to predict accurate results of certain properties of methane (Fischer, et al., 1984, Jorgensen, et al., 1996, Murad and Gubbins, 1978, Saager and Fischer, 1990, Skarmoutsos, et al., 2005, Stassen, 1999); therefore, it has been adopted in this study. Saager and Fischer (SF) (Saager and Fischer, 1990) were able to predict quite accurate simple thermodynamic data of liquid methane using the interaction parameter values $149.9 \mathrm{~K}$ and $3.733 \AA$ for the well-depth $(\varepsilon / k)$ and collision diameter $(\sigma)$, respectively. These values were obtained from fitting vapor pressure of methane and liquid densities. Murad and Gubbins (Murad and Gubbins, 1978) have used more complicated five-centered LJ potential to predict more accurate thermodynamic properties of methane. Tchouar $e t$ al. (Tchouar, et al., 2004) have studied the properties of liquid methane at low temperatures and over a large range of pressure using molecular dynamic simulation. They utilized a Feynman-Hibbs temperature-dependent potential form which lead to more accurate thermodynamic properties. None of the above-mentioned studies have used temperature-dependent interaction parameters (TDIP) model in their simulations. In a previous investigation (Al-Matar, et al., 2008, Al-Matar, et al., 2015), it has been shown that the usage of TDIP yields to more accurate vapor-liquid equilibrium properties for Argon. In this study, the vapor-liquid equilibrium of methane will be predicted using TDIP and compared with the phase diagram obtained using TIIP, and other modified parameters in the literature.

\section{Materials and Methods}

\subsection{Intermolecular potential}

The Lennard-Jones intermolecular model is used to represent the pair interaction between methane molecules $i$ and $j$ separated by the distance $r_{i j}$ :

$u\left(r_{i j}\right)=4 \varepsilon_{i j}\left[\left(\frac{\sigma_{i j}}{r_{i j}}\right)^{12}-\left(\frac{\sigma_{i j}}{r_{i j}}\right)^{6}\right]$

The second virial coefficient $B$ was utilized to determine the models of the interaction parameters, $\varepsilon_{i j}$ and $\sigma_{i j}$, as a function of temperature. An optimization process was carried out aiming to minimize the least-squares of the errors between the experimental values of the second virial coefficient (Dymond and Smith, 1980) and those calculated using the equation:

Received on October 18, 2018, accepted on December 9, 2018. Correspondence concerning this article should be addressed to Ibrahim Suleiman (Email address: Dr.Ibrahim.Suleiman@bau.edu.jo or Dr.Ibrahim.Suleiman@ gmail.com). ORCiD ID of Ibrahim Suleiman: https://orcid.org/0000-0002-7311-9227 
$B_{\text {classical }}=-2 \pi N_{A} \int_{0}^{\infty}\left[e^{-u(r) / k T}-1\right] r^{2} d r$

where $N_{A}$ is the Avogadro's number and $k$ is the Boltzmann constant. This nonlinear optimization problem was solved using the Marquardt-Levenberg (Marquardt, 1963, Press, et al., 1992) method by minimizing the root mean square deviation (RMSD) value, given by:

$R M S D=\sqrt{\frac{1}{M} \sum_{i=1}^{M}\left(B_{i, \exp }-B_{i, c a l}\right)^{2}}$

where $\mathrm{M}$ is the number of observations, $B_{i, \text { exp }}$ and $B_{i, c a l}$ are the experimental and calculated second virial coefficient for the $i$ th observation, respectively. The integral was evaluated using a 21-point Gauss-Kronrod quadrature to estimate the integral and the associated errors

Only two-parameter relationships between the independent variable, the temperature, and the dependent variables $\varepsilon / k$ and $\sigma$ were attempted. These relationships involved combinations of linear, reciprocal, exponential and reciprocal-exponential terms. Only models with parameters that cause $\sigma$ to increase and $\varepsilon / k$ to decrease with temperature were considered feasible (Al-Matar, et al., 2008, Al-Matar, et al., 2015). The feasible models were subjected to a model discrimination process based on calculating posterior probability (Marquardt, 1963). Table 1 shows the values of TIIP in the literature and the chosen model of TDIP.

Within the temperature range of interest for methane 120-190 K, used in the simulations which were carried out, the value of $\sigma$ changes between 3.74 and $3.91 \AA$, and the value of $\varepsilon / k$ changes in the range $164.75-157.30 \mathrm{~K}$. While the range of $\sigma$ is in general agreement with available temperature-independent values, the values of $\varepsilon / k$ are higher by $6.1-11.2 \%$.

Table 1 Temperature independent values and Temperature dependent models of the interaction parameters.

\begin{tabular}{lll}
\hline & $\varepsilon / k(\mathrm{~K})$ & \multicolumn{1}{c}{$\sigma(\AA)$} \\
\hline $\begin{array}{l}\text { Temperature Independent Value } \\
\text { (Stassen, 1999) }\end{array}$ & 148.2 & 3.82 \\
& & \\
Temperature dependent Model & 144.54 & 4.199 \\
& $+2425.014 / T$ & $-55.168 / T$
\end{tabular}

\subsection{Simulation details}

Gibbs ensemble is utilized to simulate the coexistence of vapor and liquid phases using systems of 500 atoms. All simulations were carried out with a spherical potential truncation for separations greater than $2.5 \sigma$ and tail corrections included. The vapor-liquid simulations were started using two boxes with simple cubic lattice. The number of atoms was equally distributed between the two boxes. The temperature of the entire system is maintained constant and surface effects are avoided by placing each box at the center of a periodic array of identical boxes. The simulations were carried out with 200 equilibration and 2000 production cycles. The type of Monte Carlo moves were selected at random according to the following probabilities: 0.9089 translation, 0.0909 particle swap and 0.0002 volume exchange with one volume exchange per cycle.

The results obtained from the simulations include energy, pressure, density, chemical potential and number of atoms for both vapor and liquid phases. The uncertainties in the ensemble averages were calculated by dividing the post-equilibrium results into ten blocks then taking the grand average (Frenkel and Smit, 1996). The code employed in this work is developed in-house using object-oriented programming in FORTRAN-90 and it is available upon request.

\subsection{Critical constants}

Gibbs ensemble simulations become unstable near the critical region due to the small difference of the free energy between the two phases. According to the scaling law, in the case of Gibbs ensemble simulation, the critical temperature is evaluated by the calculated $\rho-T$ coexistence data (Panagiotopoulos, 1994):

$\rho_{L}-\rho_{V}=b\left(T_{c}-T\right)^{\gamma}$

Where $\gamma$ is the non-classical 3D Ising critical exponent $(\gamma=0.325)$. The parameter $b$ and the critical temperature $T_{c}$ will be obtained from the fit. Subsequently, the critical density $\rho_{c}$ can be determined using a fit based on the law of rectilinear diameters (Panagiotopoulos, 1994):

$\frac{\left(\rho_{L}+\rho_{V}\right)}{2}=\rho_{c}+A\left(T_{c}-T\right)$

where the parameter $A$ and the critical density $\rho_{c}$ will be evaluated from the fit. 


\section{Results and Discussion}

The second virial coefficient data was fitted using TIIP as well as TDIP forms. Figure 1 shows the residuals for the second virial coefficient data of methane using the values available in the literature $\sigma=3.82 \AA$ and $\varepsilon / k=148.2 \mathrm{~K}$ (Stassen, 1999), and fitted temperature-dependent models, $\sigma=4.199-55.168 / T$ and $\varepsilon / k=144.54+2425.014 / T$. Using TDIP the model yield to closer values of the second virial coefficient than TIIP form. The improvement appears in Figure 1 as the difference between experimental and calculated values decreases in temperature-dependent case and gets closer to the zero line than the temperature-independent case. However, similar to the observation in a previous work (Al-Matar, et al., 2008, Al-Matar, et al., 2015), the residuals still show systematic trends suggesting that the temperature-independent parameters are inadequate to describe the behavior of the second virial coefficient over a wide temperature range.

Figure 2 compares the simulation results of the vapor-liquid coexistence curve of this work using a TDIP model and the simulation values obtained using TIIP taken from the literature. It also shows the simulation results of Skarmoutsos (Skarmoutsos, et al., 2005) using Saager-Fischer (Fischer, et al., 1984, Saager and Fischer, 1990) model of LJ potential as well as the experimental data (Kleinrahm, et al., 1988). A summary of the results using the TDIP model is given in Table 2.

Table 2 Coexistence energies, pressures, and densities, of pure methane for different temperatures at a total number of atoms $\mathrm{N}=500$

\begin{tabular}{|c|c|c|c|c|c|c|}
\hline \multirow[b]{2}{*}{ Temperature (K) } & \multicolumn{2}{|c|}{ Energy $(\mathrm{J} / \mathrm{mol})$} & \multicolumn{2}{|c|}{ Pressure (bar) } & \multicolumn{2}{|c|}{ Density $\left(\mathrm{g} / \mathrm{cm}^{3}\right)$} \\
\hline & Liquid & Vapor & Liquid & Vapor & Liquid & Vapor \\
\hline 120 & -8331.7 & -132.5 & 9.1 & 1.34 & 0.428 & $2.391 \times 10^{-3}$ \\
\hline 140 & -7398.3 & -184.8 & 1.0 & 3.15 & 0.372 & $4.782 \times 10^{-3}$ \\
\hline 160 & -6506.4 & -404.0 & 1.7 & 9.30 & 0.324 & $1.339 \times 10^{-2}$ \\
\hline 180 & -5612.2 & -867.8 & 21.7 & 22.0 & 0.279 & $3.347 \times 10^{-2}$ \\
\hline 190 & -5205.9 & -1797.4 & 40.93 & 32.44 & 0.259 & $6.873 \times 10^{-2}$ \\
\hline
\end{tabular}

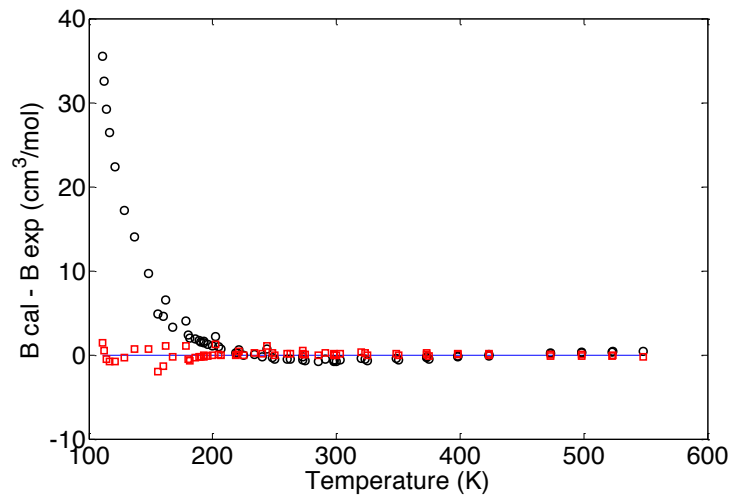

Fig. 1 Residuals between experimental second virial coefficient and calculated second virial coefficient. ( $\bigcirc$ Temperature independent case, $\square$ Temperature dependent case).

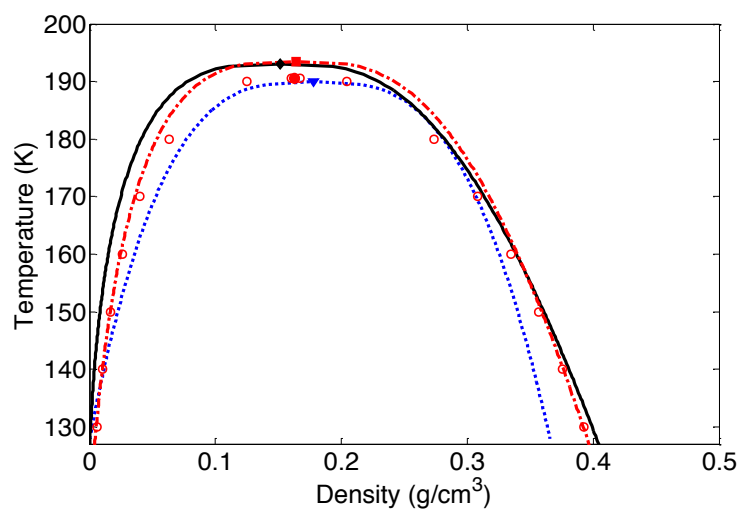

Fig. 2 Vapor-liquid phase diagram curve for methane. ( $\bigcirc)$ Experimental data(Kleinrahm, et al., 1988), (......) temperature independent curve, (-.--.-.)Skarmoutsos-Saager-Fischer model (Skarmoutsos, et al., 2005)-, (-) Temperature dependent curve. Experimental critical point, $\boldsymbol{\nabla}$ temperature independent critical point, Skarmoutsos-Saager-Fischer model-critical point, $\bullet$ temperature dependent critical point.

The results of this work obtained using TDIP are closer to the experimental data than the values produced using TIIP. The RMSD values were calculated to be $5.6 \times 10^{-2}$ and $1.07 \mathrm{~g} / \mathrm{cm}^{3}$ for simulations using TDIP and TIIP, respectively (see Table 3). The gain in accuracy as measured by the RMSD is, therefore, about 18 folds compared to the TIIP values in the literature. The deviation between the experimental values (Kleinrahm, et al., 1988) of the critical density of methane and the predicted results using TDIP and TIIP in the literature are $-7.4 \%$ and $9.2 \%$, respectively. The vapor-liquid coexistence curve of this work, however, is close to the predicted line by Skarmoutsos et al., who adopted the Saager-Fischer (SF) model of LJ potential (Skarmoutsos, et al., 2005). The RMSD obtained using the results of Skarmoutsos et al., (Skarmoutsos, et al., 2005) was found to be $4.1 \times 10^{-2} \mathrm{~g} / \mathrm{cm}^{3}$. The RMSD values were calculated from the experimental data (Kleinrahm, et al., 1988) of the density in the temperature range 120-190 K. Saager-Fisher model of $L J$ potential was obtained from fitting vapor pressures and liquid densities; and therefore, it is anticipated that the simulations of Skarmoutsos (Skarmoutsos, et al., 2005) produce more accurate values in the liquid region. This procedure has also enhanced the prediction of the critical density $\left(\rho_{c}\right)$. The values of $\rho_{c}$ are calculated to be $0.151 \mathrm{~g} / \mathrm{cm}^{3}$, with $6.9 \%$ deviation from the experimental value, and $0.162 \mathrm{~g} / \mathrm{cm}^{3}$, with $0.5 \%$ deviation from the experimental value, using TDIP and TIIP of SF model(Skarmoutsos, et al., 2005), respectively. On the other hand, the predicted critical temperature using TDIP $\left(T_{c}=193.1 \mathrm{~K}\right)$ was more accurate than reported temperature by Skarmoutsos et al. $\left(T_{c}=194.3 \mathrm{~K}\right)$. 
Figure 3 illustrates the vapor pressure versus temperature curves obtained from vapor phase results using TDIP and compares it with the experimental vapor pressure data, as fitted by the Antoine equation, for methane. The values of RMSD in vapor pressure of methane using TIIP, TDIP and TIIP-SF model are shown in Table 4. The values of RMSD obtained from this work is the highest compared to the results using TIIP and SF model. It is anticipated that using the vapor pressures to fit the LJ potential in the SF model enhances the results of vapor pressure predictions. This is due to fitting the parameters to the vapor pressure of methane. However, compared to a previous investigation (Al-Matar, et al., 2008, Al-Matar, et al., 2015), the resulting RMSD value in vapor pressure of argon, 6.21 bar is close to the current RMSD result of methane, 6.57 bar. This indicates that the error in our simulation in vapor pressure has a similar trend in both

Table 3 Comparison between critical densities and temperatures for different studies studies.

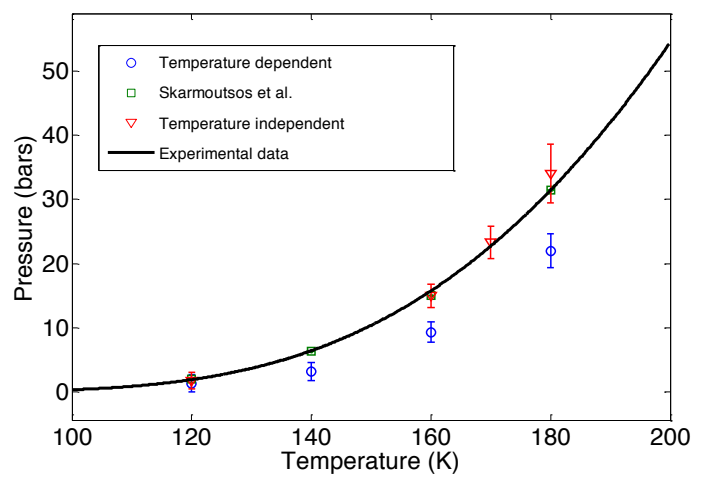

Table 4 RMSD in vapor pressure of methane for cases of TIIP, TIIP-SF model and TDIP

\begin{tabular}{lc}
\hline & $R M S D$ (bar) \\
\hline TIIP & 0.742 \\
TIIP-SF model (Skarmoutsos, et al., 2005) & 0.830 \\
TDIP (this work) & 6.568 \\
\hline
\end{tabular}

Fig. 3 Vapor pressure of methane versus temperature for different systems.

\section{Conclusions}

Two parameter models of the interaction parameters, $\sigma=4.199-55.168 / T$ and $\varepsilon / k=144.54+2425.014 / T$ in LJ potential were used to simulate the vapor-liquid phase diagram of methane. It was shown that using TDIP improves the simulated vapor-liquid coexistence curve of methane modeled as an LJ fluid. Adopting TDIP in the simulations reduces the RMSD of density by $94.7 \%$ relative to the values generated using TIIP. However, the predicted critical values were somewhat mixed; as the calculated critical temperature was improved, while the opposite was true for the evaluated critical density. The results of this study are close to the simulations of TIIP using SF model, which has been obtained by fitting the vapor pressure and liquid densities of methane. Their results show closer values to the experimental data notably in the liquid phase region.

\section{Nomenclature}

$\begin{array}{lll}A & =\text { Ising scaling law parameter } & {[-]} \\ b & =\text { Ising scaling law parameter } & {[-]} \\ B_{\text {classical }} & =\text { Second virial coefficient } & {\left[\mathrm{cm}^{3} / \mathrm{mol}\right]} \\ B_{i, \text { cal }} & =\text { Calculated second virial coefficient } & {[-]} \\ B_{i, \text { exp }} & =\text { Experimental second virial coefficient } & {[-]} \\ k & =\text { Boltzmann Constant } & {[-]} \\ \mathrm{LJ} & =\text { Lennard-Jones } & {[-]} \\ M & \text { =Number of values } & {[-]} \\ \mathrm{N} & =\text { Number of atoms } & {[-]} \\ N_{A} & =\text { Avogadro's Numbers } & {[-]} \\ r & =\text { Distance } & {[\AA]} \\ R M S D & =\text { Root mean square deviation } & {[-]} \\ \mathrm{SF} & =\text { Saager-Fischer } & {[-]} \\ T & =\text { Temperature } & {[\mathrm{K}]} \\ T_{c} & =\text { Critical temperature } & {[\mathrm{K}]} \\ \mathrm{TDIP} & =\text { Temperature dependent interaction parameters } & {[-]} \\ \text { TIIP } & =\text { Temperature independent interaction parameters } & {[-]} \\ u & =\text { Interatomic Potential } & {[\mathrm{J}]} \\ \varepsilon_{i j} & =\text { Well-depth } & {[\mathrm{cm}]}\end{array}$


$\gamma \quad=$ The non-classical 3D Ising critical exponent

$\rho_{c} \quad=$ Critical density

$\rho_{L} \quad=$ Density of liquid phase

$\rho_{V} \quad=$ Density of vapor phase

$\sigma_{i j} \quad=$ Collision Diameter $[\gamma=0.325]$

$\left[\mathrm{g} / \mathrm{cm}^{3}\right]$

$\left[\mathrm{g} / \mathrm{cm}^{3}\right]$

$\left[\mathrm{g} / \mathrm{cm}^{3}\right]$

$[\AA]$

\section{References}

Al-Matar, A., Tobgy, A., and Suleiman, I. "The phase diagram of the Lennard-Jones fluid using temperature-dependent interaction parameters", Molec. Sim., 34, 289-294 (2008).

Al-Matar, A., Tobgy, A., Suleiman, I., and Al-Faiad, M. "Improving monte-carlo and molecular dynamics simulation outcomes using temperature-dependent interaction parameters: The case of pure LJ fluid", Int. J. of Comp. Meth., 12 , 1550003-1550004-14 (2015)

Betaouaf, F., Cailliez, F., Rousseau, B., and Ould-Kaddour, F. "Molecular simulation of the thermodynamics, structural and transport properties of the liquid binary mixture methane+nitrogen", J. of Molec. Liqu., 200, 298-304 (2014).

Dymond, J., and Smith, E. "The Virial Coefficients of Pure Gases and Mixtures", Second corrected printing, John Wiley and Sons, New York (1980)

Fischer, J., Lustig, R., Breitenfelder-Manske, H., and Lemming, W. "Influence of intermolecular potential parameters on orthobaric properties of fluids consisting of spherical and linear molecules", Molec. Phys., 52, 485-497 (1984).

Frenkel, D., and Smit, B. "Understanding molecular simulation: From algorithms to applications", Academic Press, London, (1996).

Jorgensen, W., Maxwell, D., and Tirado-Rives, J. "Development and testing of the OPLS all-atom force field on conformational energetics and properties of organic liquids", J. of the Amer. Chem. Soc., 118, 11225-11236 (1996).

Kleinrahm, R., Duschek, W., Wagner, W., and Jaeschke, M. "Measurement and correlation of the (pressure, density, temperature) relation of methane in the temperature range from $273.15 \mathrm{~K}$ to $323.15 \mathrm{~K}$ at pressures up to $8 \mathrm{MPa}$ ", The J. of Chem. Therm., 20, 621-631 (1988).

$\mathrm{Li}, \mathrm{Y}$., Yu, Y., Zheng, Y., and Li, J. "Vapor-liquid equilibrium properties for confined binary mixtures involving $\mathrm{CO}_{2}, \mathrm{CH}_{4}$, and $\mathrm{N}_{2}$ from Gibbs ensemble Monte Carlo simulations", Sci. China-Chem., 55, 1825-1831 (2012).

Marquardt, D., "An algorithm for least-squares estimation of nonlinear parameters", J. Soc. Ind. Appl. Math., 11, 431-441 (1963).

Murad, S., and Gubbins, K. "Computer modeling of matter", ACS Symp. Ser., 86, 62-71 (1978)

Nie, X., Zhao, L., Deng, S., Su, W., and Zhang, Y. "A review of molecular simulation applied in vapor-liquid equilibria (VLE) estimation of thermodynamic cycles", J. of Molec. Liquids, 264, 652-674 (2018).

Panagiotopoulos, A., "Molecular simulation of phase coexistence: Finite-size effects and determination of critical parameters for two- and three-dimensional Lennard-Jones fluids", Int. J. of Therm., 15, 1057-1072 (1994).

Petropoulou, E., Voutsas, E., Westman, S., Austegard, A., Stang, H., and Løvseth S. "Vapor-liquid equilibrium of the carbon dioxide/methane mixture at three isotherms", Flu. Phase Equi., 462, 44-58 (2018).

Press, W., Teukolsky, S., Vetterling, W., and Flannery, B. "Numerical Recipes", Cambridge University Press, New York (1992)

Saager, B., and Fischer J. "Predictive power of effective intermolecular pair potentials: MD simulation results for methane up to 1000 MPa", Flu. Phase Equ., 57, 35-46 (1990).

Skarmoutsos, I., Kampanakis, L., and Samios, J. "Investigation of the vapor-liquid equilibrium and supercritical phase of pure methane via computer simulations", J. of Molec. Liqu., 117, 33-41 (2005).

Stassen, H. "On the pair potential in dense fluid methane". J. of Molec. Struc., THEOCHEM, 464, 107-119 (1999).

Tchouar, N., Ould-Kaddour, F., and Levesque, D. "Computation of the properties of liquid neon, methane, and gas helium at low temperature by the FeynmanHibbs approach", J. of Chem. Phy., 121, 7326-7331 (2004).

Ungerer, P., Nieto-Draghi, C., Rousseau, B., Ahunbay, G., and Lachet, V. "Molecular simulation of the thermophysical properties of fluids: From understanding toward quantitative predictions", J. of Molec. Liqu., 134, 71-89 (2007).

Uribe-Vargas, V., and Trejo, A. "Vapor-liquid equilibrium of methane and methane plus nitrogen and an equimolar hexane plus decane mixture under isothermal conditions", Flu. Phase Equ., 238, 95-105 (2005).

Vrabec, J., and Fischer, J."Vapor-liquid equilibria of binary mixtures containing methane, ethane, and carbon dioxide from molecular simulation", Int. J. of Thermophysics, 17, 889-908 (1996). 